\title{
Global existence and nonexistence for a class of parabolic systems with time-dependent coefficients
}

\author{
Anyin Xia ${ }^{1,2}$, Xianxiang $\mathrm{Pu}^{3}$ and Shan $\mathrm{Li}^{4^{*}}$
}

\section{"Correspondence:}

lishan@scu.edu.cn

${ }^{4}$ Business School, Sichuan

University, Chengdu, 610065, P.R.

China

Full list of author information is

available at the end of the article (c) 2016 Xia et al. This article is distributed under the terms of the Creative Commons Attribution 4.0 International License (http://creativecommons.org/licenses/by/4.0/), which permits unrestricted use, distribution, and reproduction in any medium, provided you give appropriate credit to the original author(s) and the source, provide a link to the Creative Commons license, and indicate if changes were made.

\begin{abstract}
In this paper, we study the global existence and nonexistence to the nonnegative solution of a class of parabolic systems with time-dependent coefficients. More precisely, the existence of a global solution is established via the standard comparison principle. Furthermore, we establish a blow-up solution and obtain both upper and lower bounds for the maximum blow-up time under some appropriate hypotheses.
\end{abstract}

MSC: 35K20; 35K55; 35K65; 80M35

Keywords: parabolic systems; degenerate systems; global existence; blow-up; the maximum blow-up time

\section{Introduction and main results}

This paper is concerned with the following parabolic systems with time dependent coefficients:

$$
\begin{cases}u_{t}=\Delta u^{m}+f_{1}(t) v^{p}, & (x, t) \in \Omega \times(0, T), \\ v_{t}=\Delta v^{n}+f_{2}(t) u^{q}, & (x, t) \in \Omega \times(0, T), \\ u(x, t)=v(x, t)=0, & (x, t) \in \partial \Omega \times(0, T), \\ u(x, 0)=u_{0}(x), \quad v(x, 0)=v_{0}(x), & x \in \Omega .\end{cases}
$$

Here $\Omega \subset \mathbb{R}^{N}$ is a bounded domain with smooth boundary $\partial \Omega, m, n>1, p, q>0, f_{1}(t)$, and $f_{2}(t)$ are positive bounded continuous functions with $f_{1}(t) \leq \bar{k}_{1}, f_{2}(t) \leq \bar{k}_{2}$ for any $t \geq 0$. The initial data $u_{0}(x), v_{0}(x)$ are nontrivial nonnegative continuous functions and satisfy the compatibility conditions $u_{0}(x)=v_{0}(x)=0$ on the boundary $\partial \Omega$.

Global existence and singularity analyses of the solutions to the nonlinear parabolic equation have been investigated extensively [1] and [2]. It is well known that the solutions of parabolic problems may remain bounded for all time, or blow up in a finite time. When blow-up occurs in a finite time $T$, the evaluation of maximal blow-up time $T$ is of great practical interest. The first purpose in this paper is to investigate the sufficient conditions to the global existence or nonexistence of the classical solution to the boundary value problem (1.1). Furthermore, we will investigate the solution which blows up in a finite time and estimate the life span of the singular solution. 
One of the motivations to investigate the singular solution comes from [3], in which the single parabolic equation in the linear diffusion case $(m=1)$ has been considered. However, the degenerate diffusion and the structure here make the present problem more complicated and show more essential difficulties here. We would like to recall some results on blow-up solutions to the degenerate parabolic equations and system in [4-8] and the references therein. We also would like to mention some results on the global existence and nonexistence of the classical solution of several similar mathematical models in [2, 4-17].

First of all, we give the global existence of the classical solution to the boundary value problem (1.1) as follows.

Theorem 1.1 If $p q<m n$, then every classical solution to the initial-boundary value problem (1.1) is global.

Second, the result on the blow-up solution to the boundary value problem (1.1) is established in the next theorem.

Theorem 1.2 Assume $\underline{k}:=\min \left\{\inf f_{1}(t), \inf f_{2}(t)\right\}>0$. If $p q>m n$, then the classical solution to the initial-boundary value problem (1.1) blows up in finite time $T$ for large data $u_{0}(x)$, $v_{0}(x)$.

In the following theorem, we give the upper bound for the blow-up time as long as blowup occurs.

Theorem 1.3 Assume $\underline{k}:=\min \left\{\inf f_{1}(t), \inf f_{2}(t)\right\}>0$. If $q \geq m, p \geq n, \underline{k}>\lambda_{1}$, then the classical solution to the initial-boundary value problem (1.1) blows up in finite time $T$ for large data $u_{0}(x), v_{0}(x)$, where $\lambda_{1}$ is the first eigenvalue to the following problem:

$$
\begin{cases}\Delta \phi+\lambda \phi=0, & x \in \Omega \\ \phi=0, & x \in \partial \Omega\end{cases}
$$

with $\phi \geq 0$ and $\int_{\Omega} \phi d x=1$. Moreover, there exists $a T_{0}>0$, which depends on $p, q, m, n, \underline{k}$, and the initial data, such that $T \leq T_{0}$.

Remark 1.1 Indeed, the idea to show Theorem 1.3 is based on the method in [15] to obtain an estimate to the life span. However, there are still some essential differences and difficulties. One is that the system considered in the present paper is degenerate and quasilinear, and the system in [15] is semilinear. As we know, there are many essential difficulties to extend the technique to the degenerate system. The method in [15] cannot be applied to the quasilinear case directly. Here we borrowed the idea and modified the technique in [15] to deal with the degenerate system and obtain the estimates of the life span to a degenerate, quasilinear system. The second one is that we can deal with the critical case in this paper, which is one of the main differences from the results in [15].

Remark 1.2 In fact, along the proof of Theorem 1.3, we can conclude that the solution blows up in finite time for the critical case $p=n$ and $q=m$.

Finally, we give a lower estimate to the maximum blow-up time $T$. 
Theorem 1.4 Suppose that $\Omega$ is a convex domain in $\mathbb{R}^{3}$ and $(u, v)$ blows up in finite time $T$; there exists a positive constant $\underline{T}_{0}$ such that $T \geq \underline{T}_{0}$, where $\underline{T}_{0}$ depends only on $m, n, p$, $q, f_{1}, f_{2}$, and the initial data.

The remainder of this paper is organized as follows. The global existence of the solution to the problem (1.1) is established in Section 2, by the standard comparison argument. In Section 3, we show that the classical solution to the problem (1.1) will blow up in a finite time for some sufficiently large initial data. In the final section, with the aid of some differential inequality, we will establish both lower and upper estimates to the maximal blow-up time.

\section{Global solution for the problem (1.1)}

In this section, we focus on the global solution to the problem (1.1). First, set

$$
A=\left(\begin{array}{cc}
m & -p \\
-q & n
\end{array}\right) \quad \text { and } \quad l=\left(\begin{array}{l}
l_{1} \\
l_{2}
\end{array}\right) \text {. }
$$

In the following, we will introduce some lemmas which play important roles in the following proof. We would like to refer to [9] and [4] for the proof.

Lemma 2.1 If $p q<m n$, then there exist two positive constants $l_{1}, l_{2}$, such that $A l=(1,1)^{T}$. Moreover, $A(c l)>(0,0)^{T}$ for any $c>0$.

Lemma 2.2 If $p q>m n$, then there exist two positive constants $l_{1}, l_{2}$, such that $A l<(0,0)^{T}$. Moreover, $A(c l)<(0,0)^{T}$ for any $c>0$.

Next, we will give the proof of Theorem 1.1 as follows.

Proof Here, we construct some super-solution to the problem, which is bounded for any $T>0$. Let $\varphi(x)$ be the solution of the following elliptic problem:

$$
\begin{cases}-\Delta \varphi(x)=1, & x \in \Omega, \\ \varphi(x)=0, & x \in \partial \Omega .\end{cases}
$$

Denote $C=\max _{\bar{\Omega}} \varphi(x)$. Namely, $0 \leq \varphi(x) \leq C$.

We define the functions $\bar{u}(x, t)$ and $\bar{v}(x, t)$ as

$$
\bar{u}=(K(\varphi(x)+1))^{l_{1}}, \quad \bar{v}=(K(\varphi(x)+1))^{l_{2}},
$$

where $l_{1}, l_{2}$ satisfy $m l_{1}<1, n l_{2}<1$, and $K>0$ will be fixed later. Clearly, $(\bar{u}, \bar{v})$ is bounded for any $t>0$ and $\bar{u} \geq K^{l_{1}}, \bar{v} \geq K^{l_{2}}$. Thus, a series of direct computations give

$$
\begin{aligned}
\bar{u}_{t}-\Delta \bar{u}^{m} & =-m l_{1} K^{m l_{1}}\left\{\left(m l_{1}-1\right)[1+\varphi(x)]^{m l_{1}-2}|\nabla \varphi(x)|^{2}+[1+\varphi(x)]^{m l_{1}-1} \Delta \varphi(x)\right\} \\
& \geq-m l_{1} K^{m l_{1}}[1+\varphi(x)]^{m l_{1}-1} \Delta \varphi(x)=m l_{1} K^{m l_{1}}[1+\varphi(x)]^{m l_{1}-1} \\
& \geq m l_{1} K^{m l_{1}}(1+C)^{m l_{1}-1},
\end{aligned}
$$


and

$$
f_{1}(t) \bar{\nu}^{p} \leq \bar{k}_{1} \bar{\nu}^{p} \leq \bar{k}_{1}[K(1+\varphi(x))]^{p l_{2}} \leq \bar{k}_{1}[K(1+C)]^{p l_{2}} .
$$

Similarly, we have

$$
\begin{aligned}
\bar{v}_{t}-\Delta \bar{v}^{n} & =-n l_{2} K^{n l_{2}}\left\{\left(n l_{2}-1\right)[1+\varphi(x)]^{n l_{2}-2}|\nabla \varphi(x)|^{2}+[1+\varphi(x)]^{n l_{2}-1} \Delta \varphi(x)\right\} \\
& \geq n l_{2} K^{n l_{2}}(1+C)^{n l_{2}-1}
\end{aligned}
$$

and

$$
f_{2}(t) \bar{u}^{q} \leq \bar{k}_{2} \bar{u}^{q} \leq \overline{k_{2}}[K(1+\varphi(x))]^{q l_{1}} \leq \bar{k}_{2}[K(1+C)]^{q l_{1}} .
$$

Set

$$
K_{1}=\left[\frac{\bar{k}_{1}}{m l_{1}}(1+C)^{p l_{2}-m l_{1}+1}\right]^{\frac{1}{m l_{1}-p l_{2}}} \quad \text { and } \quad K_{2}=\left[\frac{\bar{k}_{2}}{n l_{2}}(1+C)^{q l_{1}-n l_{2}+1}\right]^{\frac{1}{n l_{2}-q l_{1}}} \text {. }
$$

If $p q<m n$, it follows from Lemma 2.1 that there exist positive constants $l_{1}, l_{2}$, such that $m l_{1}-p l_{2}>0, n l_{2}-q l_{1}>0$, and $m l_{1}<1, n l_{2}<1$.

Therefore, we can choose $K$ sufficiently large so that $K>\max \left\{K_{1}, K_{2}\right\}$ and

$$
[K(\varphi(x)+1)]^{l_{1}} \geq u_{0}(x), \quad[K(\varphi(x)+1)]^{l_{2}} \geq v_{0}(x) .
$$

Now, it follows from (2.3)-(2.8) that $(\bar{u}, \bar{v})$ defined by (2.2) is a positive super-solution to the problem (1.1). Hence, the comparison principle gives $(u, v) \leq(\bar{u}, \bar{v})$, which implies $(u, v)$ exists globally.

\section{Blow-up solution for the problem (1.1)}

In this section, we will discuss the blow-up solution to the problem (1.1) under some appropriate hypotheses and show Theorem 1.2.

Proof We will construct some blow-up subsolution in some subdomain of $\Omega$ in which $u, v>0$. Some ideas are borrowed from work by $\mathrm{Du}[6]$.

Let $\psi(x)$ be a nontrivial nonnegative continuous function that vanishes on $\partial \Omega$. Without loss of generality, we assume that $0 \in \Omega$ and $\psi(0)>0$. We shall construct a blow-up subsolution to complete the proof.

Set

$$
\underline{u}(x, t)=\frac{1}{(T-t)^{l_{1}}} \omega^{\frac{1}{m}}\left(\frac{|x|}{(T-t)^{\sigma}}\right), \quad \underline{v}(x, t)=\frac{1}{(T-t)^{l_{2}}} \omega^{\frac{1}{n}}\left(\frac{|x|}{(T-t)^{\sigma}}\right),
$$

with

$$
\omega(r)=\frac{R^{3}}{12}-\frac{R}{4} r^{2}+\frac{1}{6} r^{3}, \quad r=\frac{|x|}{(T-t)^{\sigma}}, 0 \leq r \leq R,
$$


where the parameters $l_{1}, l_{2}, \sigma$, and $T>0$ are be determined later. Clearly, $0 \leq \omega(r) \leq \frac{R^{3}}{12}$ and $\omega(r)$ is nonincreasing since $\omega^{\prime}(r)=\frac{r(r-R)}{2} \leq 0$. Note that

$$
\operatorname{supp} \underline{u}(\cdot, t)=\operatorname{supp} \underline{v}(\cdot, t)=B\left(0, R(T-t)^{\sigma}\right) \subset B\left(0, R T^{\sigma}\right) \subset \Omega,
$$

for sufficiently small $T>0$. Obviously, $(\underline{u}, \underline{v})$ becomes unbounded as $t \rightarrow T^{-}$at the point $x=0$.

Thus, we have

$$
\begin{aligned}
\underline{u}_{t}(x, t)-\Delta \underline{u}^{m}(x, t) & =\frac{m l_{1} \omega^{\frac{1}{m}}(r)+\sigma r \omega^{\prime}(r) \omega^{\frac{1-m}{m}}}{m(T-t)^{l_{1}+1}}+\frac{R-2 r}{2(T-t)^{m l_{1}+2 \sigma}}+\frac{(N-1)(R-r)}{2(T-t)^{m l_{1}+\sigma}} \\
& \leq \frac{l_{1}\left(\frac{R^{3}}{12}\right)^{\frac{1}{m}}}{(T-t)^{l_{1}+1}}+\frac{N R-(N+1) r}{2(T-t)^{m l_{1}+2 \sigma}}
\end{aligned}
$$

and

$$
\underline{v}_{t}(x, t)-\Delta \underline{v}^{n}(x, t) \leq \frac{l_{2}\left(\frac{R^{3}}{12}\right)^{\frac{1}{n}}}{(T-t)^{l^{2}+1}}+\frac{N R-(N+1) r}{2(T-t)^{n l_{2}+2 \sigma}}
$$

noticing that $T<1$ is sufficiently small.

Case 1. If $0 \leq r \leq \frac{N R}{N+1}$, we have $\omega(r) \geq \frac{(3 N+1) R^{3}}{12(N+1)^{3}}$, then

$$
f_{1}(t) \underline{p}^{p}(x, t)=f_{1}(t) \frac{1}{(T-t)^{p l_{2}}} \omega^{\frac{p}{n}}(r) \geq \frac{\underline{k}}{(T-t)^{p l_{2}}}\left(\frac{(3 N+1) R^{3}}{12(N+1)^{3}}\right)^{\frac{p}{n}}
$$

and

$$
f_{2}(t) \underline{u}^{q}(x, t)=f_{2}(t) \frac{1}{(T-t)^{q l_{1}}} \omega^{\frac{q}{m}}(r) \geq \frac{\underline{k}}{(T-t)^{q l_{1}}}\left(\frac{(3 N+1) R^{3}}{12(N+1)^{3}}\right)^{\frac{q}{m}} .
$$

Hence,

$$
\underline{u}_{t}(x, t)-\Delta \underline{u}^{m}(x, t)-f_{1}(t) \underline{v}^{p}(x, t) \leq \frac{l_{1}\left(\frac{R^{3}}{12}\right)^{\frac{1}{m}}}{(T-t)^{l_{1}+1}}-\frac{\underline{k}}{(T-t)^{p l_{2}}}\left(\frac{(3 N+1) R^{3}}{12(N+1)^{3}}\right)^{\frac{p}{n}}
$$

and

$$
\underline{v}_{t}(x, t)-\Delta \underline{v}^{n}(x, t)-f_{2}(t) \underline{u}^{q}(x, t) \leq \frac{l_{2}\left(\frac{R^{3}}{12}\right)^{\frac{1}{n}}}{(T-t)^{l_{2}+1}}-\frac{\underline{k}}{(T-t)^{q l_{1}}}\left(\frac{(3 N+1) R^{3}}{12(N+1)^{3}}\right)^{\frac{q}{m}} .
$$

Case 2. If $\frac{N R}{N+1}<r \leq R$, then

$$
\underline{u}_{t}(x, t)-\Delta \underline{u}^{m}(x, t)-f_{1}(t) \underline{v}^{p}(x, t) \leq \frac{l_{1}\left(\frac{R^{3}}{12}\right)^{\frac{1}{m}}}{(T-t)^{l_{1}+1}}+\frac{N R-(N+1) r}{2(T-t)^{m l_{1}+2 \sigma}}
$$

and

$$
\underline{v}_{t}(x, t)-\Delta \underline{v}^{n}(x, t)-f_{2}(t) \underline{u}^{q}(x, t) \leq \frac{l_{2}\left(\frac{R^{3}}{12}\right)^{\frac{1}{n}}}{(T-t)^{l_{2}+1}}+\frac{N R-(N+1) r}{2(T-t)^{n l_{2}+2 \sigma}} .
$$


If $p q>m n$, it follows from Lemma 2.2 that there exist positive constants $l_{1}, l_{2}$, such that

$$
m l_{1}-p l_{2}<-1, \quad n l_{2}-q l_{1}<-1 \quad \text { and } \quad(m-1) l_{1}>1, \quad(n-1) l_{2}>1 .
$$

Thus, we get

$$
p l_{2}>m l_{1}+1>l_{1}+1, \quad q l_{1}>n l_{2}+1>l_{2}+1,
$$

and

$$
m l_{1}+2 \sigma>l_{1}+1, \quad n l_{2}+2 \sigma>l_{2}+1
$$

for any $\sigma>0$.

Hence, for sufficiently small $\sigma>0$ and $T>0$, (3.2) holds. Thus, (3.7)-(3.10) imply that

$$
\underline{u}_{t}(x, t)-\Delta \underline{u}^{m}(x, t)-f_{1}(t) \underline{v}^{p}(x, t) \leq 0, \quad \underline{v}_{t}(x, t)-\Delta \underline{v}^{n}(x, t)-f_{2}(t) \underline{u}^{q}(x, t) \leq 0,
$$

where $(x, t) \in B\left(0, R(T-t)^{\sigma}\right) \times(0, T)$.

Since $\psi(x)$ is a nontrivial nonnegative continuous function and $\psi(0)>0$, there exist two positive constants $\rho$ and $\varepsilon$ such that $\psi(x)>\varepsilon$ for all $x \in B(0, \rho) \subset \Omega$. Choose $T$ small enough to ensure $B\left(0, R(T-t)^{\sigma}\right) \subset B(0, \rho)$, hence $\underline{u} \leq 0, \underline{v} \leq 0$ on $\partial B\left(0, R(T-t)^{\sigma}\right) \times(0, T)$. Thanks to (3.2), it follows that $\underline{u}(x, 0) \leq \bar{M} \psi(x), \underline{v}(x, 0) \leq \bar{M} \psi(x)$ for sufficient large $\bar{M}$. By the comparison principle, we have $(\underline{u}, \underline{v}) \leq(u, v)$, provided that $u_{0}(x) \geq \bar{M} \psi(x), v_{0}(x) \geq$ $\bar{M} \psi(x)$. This implies that the solution $(u, v)$ of the problem (1.1) blows up in finite time.

\section{Upper bound to the maximal blow-up time}

In this section, we will estimate the upper bound to the maximal blow-up time under some appropriate hypotheses and show Theorem 1.3.

Proof Denote

$$
\Phi(t)=\int_{\Omega} u \phi d x, \quad \Psi(t)=\int_{\Omega} \nu \phi d x \quad \text { and } \quad F(t)=\Phi(t)+\Psi(t) .
$$

Case 1. $q>m, p>n$.

With the aid of (1.1) and (4.1), we have

$$
\Phi^{\prime}(t)=\int_{\Omega}\left[\Delta u^{m}+f_{1}(t) v^{p}\right] \phi d x \geq-\lambda_{1} \int_{\Omega} u^{m} \phi d x+\underline{k} \int_{\Omega} v^{p} \phi d x
$$

and

$$
\Psi^{\prime}(t)=\int_{\Omega}\left[\Delta v^{n}+f_{2}(t) u^{q}\right] \phi d x \geq-\lambda_{1} \int_{\Omega} v^{n} \phi d x+\underline{k} \int_{\Omega} u^{q} \phi d x .
$$

Recalling that $q>m>1, p>n>1$, and applying Hölder's and Young's inequality yield

$$
\begin{aligned}
\int_{\Omega} u^{m} \phi d x & \leq\left(\int_{\Omega} u \phi d x\right)^{\frac{q-m}{q-1}}\left(\int_{\Omega} u^{q} \phi d x\right)^{\frac{m-1}{q-1}} \\
& \leq \frac{q-m}{q-1} \int_{\Omega} u \phi d x+\frac{m-1}{q-1} \int_{\Omega} u^{q} \phi d x
\end{aligned}
$$




$$
\begin{aligned}
\int_{\Omega} v^{n} \phi d x & \leq\left(\int_{\Omega} \nu \phi d x\right)^{\frac{p-n}{p-1}}\left(\int_{\Omega} v^{p} \phi d x\right)^{\frac{n-1}{p-1}} \\
& \leq \frac{p-n}{p-1} \int_{\Omega} v \phi d x+\frac{n-1}{p-1} \int_{\Omega} v^{p} \phi d x
\end{aligned}
$$

and

$$
\int_{\Omega} u^{q} \phi d x \geq\left(\int_{\Omega} u \phi d x\right)^{q}, \quad \int_{\Omega} v^{p} \phi d x \geq\left(\int_{\Omega} v \phi d x\right)^{p} .
$$

This together with (4.1) gives the following inequality:

$$
F^{\prime}(t) \geq-C_{1} F(t)+C_{2}\left[\Phi^{q}(t)+\Psi^{p}(t)\right]
$$

where $C_{1}=\max \left\{\lambda_{1} \frac{q-m}{q-1}, \lambda_{1} \frac{p-n}{p-1}\right\}>0$ and $C_{2}=\min \left\{\underline{k}-\lambda_{1} \frac{m-1}{q-1}, \underline{k}-\lambda_{1} \frac{n-1}{p-1}\right\}>0$.

For the special case $p=q$, applying the inequality

$$
a^{p}+b^{p} \geq 2^{1-p}(a+b)^{p} \quad \text { for } p>1, a, b>0
$$

we obtain

$$
F^{\prime}(t) \geq-C_{1} F(t)+2^{1-p} C_{2} F^{p}(t)
$$

Furthermore, choosing $u_{0}(x)$ sufficiently large such that

$$
2^{1-p} C_{2} F^{p-1}(0)-C_{1}>0
$$

we conclude that $F(t)$ is increasing for any $t>0$, where

$$
F(0)=\int_{\Omega}\left(u_{0}(x)+v_{0}(x)\right) \phi d x
$$

Moreover, according to (4.8) and $p>1$, we can see that there exists a $T_{1}>0$, such that

$$
\lim _{t \rightarrow T_{1}} F(t)=+\infty
$$

and

$$
T_{1} \leq \int_{F(0)}^{+\infty} \frac{d \tau}{-C_{1} \tau+2^{1-p} C_{2} \tau^{p}}<+\infty
$$

For the case $p \neq q$, without loss of generality, we assume that $p>q$. For any $c>0$, we have

$$
\Psi^{q}(t)=\left(c \Psi^{p}(t)\right)^{\frac{q}{p}}\left(c^{-\frac{q}{p-q}}\right)^{\frac{p-q}{p}} \leq \frac{q}{p} c \Psi^{p}(t)+\frac{p-q}{p} c^{-\frac{q}{p-q}} .
$$

Let $c=\frac{p}{q}$, we have

$$
\Psi^{q} \leq \Psi^{p}+A,
$$


where $A=\frac{p-q}{p}\left(\frac{p}{q}\right)^{-\frac{q}{p-q}}$. Thus,

$$
F^{\prime}(t) \geq-A C_{2}-C_{1} F(t)+C_{2}\left[\Phi^{q}(t)+\Psi^{q}(t)\right] \geq-A C_{2}-C_{1} F(t)+2^{1-q} C_{2} F^{q}(t) .
$$

Choosing $u_{0}(x)$ sufficiently large such that

$$
2^{1-q} C_{2} F^{q}(0)-C_{1} F(0)-A C_{2}>0,
$$

we conclude that $F(t)$ is monotonic increasing for any $t>0$.

Furthermore, applying (4.14) and $q>1$, we can see that there exists a $T_{2}>0$, such that

$$
\lim _{t \rightarrow T_{2}} F(t)=+\infty
$$

and

$$
T_{2} \leq \int_{F(0)}^{+\infty} \frac{d \tau}{-A C_{2}-C_{1} \tau+2^{1-q} C_{2} \tau^{q}}<+\infty .
$$

Likewise, we can obtain similar results for the following cases; there exist $T_{k}<+\infty$ $(k=3, \ldots, 8)$, such that

$$
\begin{aligned}
& T_{3} \leq \int_{F(0)}^{+\infty} \frac{d \tau}{-C_{3} \tau+2^{1-p} C_{4} \tau^{p}} \quad \text { for Case 2. } q=m, p>n(p=q), \\
& T_{4} \leq \int_{F(0)}^{+\infty} \frac{d \tau}{-A C_{4}-C_{3} \tau+2^{1-q} C_{4} \tau^{q}} \quad \text { for Case 2. } q=m, p>n(p>q), \\
& T_{5} \leq \int_{F(0)}^{+\infty} \frac{d \tau}{-C_{5} \tau+2^{1-p} C_{4} \tau^{p}} \quad \text { for Case 3. } q>m, p=n(p=q), \\
& T_{6} \leq \int_{F(0)}^{+\infty} \frac{d \tau}{-A C_{4}-C_{5} \tau+2^{1-q} C_{4} \tau^{q}} \quad \text { for Case 3. } q>m, p=n(p>q), \\
& T_{7} \leq \int_{F(0)}^{+\infty} \frac{d \tau}{2^{1-p} C_{4} \tau^{p}} \quad \text { for Case } 4 . q=m, p=n(p=q), \\
& T_{8} \leq \int_{F(0)}^{+\infty} \frac{d \tau}{-A C_{4}+2^{1-q} C_{4} \tau^{q}} \quad \text { for Case } 4 . q=m, p=n(p>q),
\end{aligned}
$$

and

$$
\lim _{t \rightarrow T_{k}} F(t)=+\infty
$$

for any $k=3, \ldots, 8$. Here, $C_{3}=\lambda_{1} \frac{p-n}{p-1}>0, C_{4}=\underline{k}-\lambda_{1}>0$, and $C_{5}=\lambda_{1} \frac{q-m}{q-1}>0$.

Hence, denoting $T_{0}=\min _{1 \leq k \leq 8} T_{k}$, Theorem 1.3 follows immediately.

\section{Lower bound to the finite blow-up time}

In this section, we study the lower bound to the blow-up time when blow-up occurs and show Theorem 1.4.

Proof Here, suppose that $\Omega$ is a convex domain in $\mathbb{R}^{3}$, and $(u, v)$ blows up in a finite time $T(<+\infty)$. 
First, setting

$$
\Gamma(t)=\int_{\Omega} u^{2 q+m+1} d x+\int_{\Omega} v^{2 p+n+1} d x,
$$

and recalling (1.1), we have

$$
\begin{aligned}
\Gamma^{\prime}(t)= & (2 q+m+1) \int_{\Omega} u^{2 q+m}\left[\Delta u^{m}+f_{1}(t) v^{p}\right] d x \\
& +(2 p+n+1) \int_{\Omega} v^{2 p+n}\left[\Delta v^{n}+f_{2}(t) u^{q}\right] d x \\
= & -\frac{(2 q+m+1)(2 q+m)}{(q+m)^{2}} \int_{\Omega}\left|\nabla u^{q+m}\right|^{2} d x+(2 q+m+1) f_{1}(t) \int_{\Omega} u^{2 q+m} v^{p} d x \\
& -\frac{(2 p+n+1)(2 p+n)}{(p+n)^{2}} \int_{\Omega}\left|\nabla v^{p+n}\right|^{2} d x+(2 p+n+1) f_{2}(t) \int_{\Omega} v^{2 p+n} u^{q} d x .
\end{aligned}
$$

Thus, Hölder's inequality implies that

$$
\begin{aligned}
& f_{1}(t) \int_{\Omega} u^{2 q+m} v^{p} d x \\
& \leq f_{1}(t)\left(\int_{\Omega} u^{2(2 q+m)} d x\right)^{\frac{1}{2}}\left(\int_{\Omega} v^{2 p} d x\right)^{\frac{1}{2}} \\
& \quad \leq f_{1}(t)\left(\int_{\Omega} u^{6(q+m)} d x\right)^{\frac{2 q+m-1}{2(4 q+5 m-1)}}\left(\int_{\Omega} u^{2 q+m+1} d x\right)^{\frac{q+2 m}{4 q+5 m-1}}\left(\int_{\Omega} v^{2 p} d x\right)^{\frac{1}{2}} .
\end{aligned}
$$

Applying Sobolev's inequality (see [1]) in $\mathbb{R}^{3}$,

$$
\left(\int_{\Omega} \xi^{6} d x\right)^{\frac{1}{6}} \leq 4^{\frac{1}{3}} 3^{-\frac{1}{2}} \pi^{-\frac{2}{3}}\left(\int_{\Omega}|\nabla \xi|^{2} d x\right)^{\frac{1}{2}}
$$

we can obtain

$$
\int_{\Omega} u^{6(q+m)} d x \leq \frac{16}{27 \pi^{4}}\left(\int_{\Omega}\left|\nabla u^{q+m}\right|^{2} d x\right)^{3} .
$$

Substituting (4.4) into (4.3) yields

$$
\begin{aligned}
f_{1}(t) & \int_{\Omega} u^{2 q+m} v^{p} d x \\
\leq & f_{1}(t)\left(\frac{2 \sqrt[3]{2}}{3 \pi \sqrt[3]{\pi}} \varepsilon_{1} \int_{\Omega}\left|\nabla u^{q+m}\right|^{2} d x\right)^{\frac{3(2 q+m-1)}{2(4 q+5 m-1)}} \\
& \times\left\{\varepsilon_{1}^{-\frac{3(2 q+m-1)}{2 q+7 m+1}}\left(\int_{\Omega} u^{2 q+m+1} d x\right)^{\frac{2 q+4 m}{2 q+7 m+1}}\left(\int_{\Omega} v^{2 p} d x\right)^{\frac{4 q+5 m-1}{2 q+7 m+1}}\right\}^{\frac{2 q+7 m+1}{2(4 q+5 m-1)}} \\
\leq & \frac{\sqrt[3]{2}(2 q+m-1)}{\pi \sqrt[3]{\pi}(4 q+5 m-1)} \varepsilon_{1} \int_{\Omega}\left|\nabla u^{q+m}\right|^{2} d x \\
& +\frac{2 q+7 m+1}{2(4 q+5 m-1)} \varepsilon_{1}^{-\frac{3(2 q+m-1)}{2 q+7 m+1}}\left(\int_{\Omega} u^{2 q+m+1} d x\right)^{\frac{2 q+4 m}{2 q+7 m+1}}\left[f_{1}^{2}(t) \int_{\Omega} v^{2 p} d x\right]^{\frac{4 q+5 m-1}{2 q+7 m+1}}
\end{aligned}
$$




$$
\begin{aligned}
\leq & \frac{\sqrt[3]{2}(2 q+m-1)}{\pi \sqrt[3]{\pi}(4 q+5 m-1)} \varepsilon_{1} \int_{\Omega}\left|\nabla u^{q+m}\right|^{2} d x \\
& +\frac{2 q+7 m+1}{2(4 q+5 m-1)} \varepsilon_{1}^{-\frac{3(2 q+m-1)}{2 q+7 m+1}}\left(\int_{\Omega} u^{2 q+m+1} d x\right)^{\frac{2 q+4 m}{2 q+7 m+1}} f_{1}(t)^{\frac{2(4 q+5 m-1)}{2 q+7 m+1}} \\
& \times\left(\int_{\Omega} v^{2 p+n+1} d x\right)^{\frac{2 p(4 q+5 m-1)}{(2 q+7 m+1)(2 p+n+1)}}|\Omega|^{1-\frac{2 p}{2 p+n+1}}
\end{aligned}
$$

where $\varepsilon_{1}=\frac{\pi \sqrt[3]{\pi}(2 q+m)(4 q+5 m-1)}{\sqrt[3]{2}(q+m)^{2}(2 q+m-1)}$.

Similarly, one has

$$
\begin{aligned}
f_{2}(t) & \int_{\Omega} v^{2 p+n} u^{q} d x \\
\leq & \frac{\sqrt[3]{2}(2 p+n-1)}{\pi \sqrt[3]{\pi}(4 p+5 n-1)} \varepsilon_{2} \int_{\Omega}\left|\nabla v^{p+n}\right|^{2} d x \\
& +\frac{2 p+7 n+1}{2(4 p+5 n-1)} \varepsilon_{2}^{-\frac{3(2 p+n-1)}{2 p+7 n+1}}\left(\int_{\Omega} v^{2 p+n+1} d x\right)^{\frac{2 p+4 n}{2 p+7 n+1}} f_{2}(t)^{\frac{2(4 p+5 n-1)}{2 p+7 n+1}} \\
& \times\left(\int_{\Omega} u^{2 q+m+1} d x\right)^{\frac{2 q(4 p+5 n-1)}{(2 p+7 n+1)(2 q+m+1)}}|\Omega|^{1-\frac{2 q}{2 q+m+1}}
\end{aligned}
$$

where $\varepsilon_{2}=\frac{\pi \sqrt[3]{\pi}(2 p+n)(4 p+5 n-1)}{\sqrt[3]{2}(p+n)^{2}(2 p+n-1)}$.

Substituting (5.5) and (5.6) into (5.2), one has

$$
\begin{aligned}
\Gamma^{\prime}(t) \leq & k(t)\left[\left(\int_{\Omega} u^{2 q+m+1} d x\right)^{\mu_{1}}\left(\int_{\Omega} v^{2 p+n+1} d x\right)^{\mu_{2}}\right. \\
& \left.+\left(\int_{\Omega} v^{2 p+n+1} d x\right)^{\mu_{3}}\left(\int_{\Omega} u^{2 q+m+1} d x\right)^{\mu_{4}}\right] \\
= & k(t)\left[\left(\int_{\Omega} u^{2 q+m+1} d x\right)^{\frac{\mu_{1}}{\mu_{1}+\mu_{2}}}\left(\int_{\Omega} v^{2 p+n+1} d x\right)^{\frac{\mu_{2}}{\mu_{1}+\mu_{2}}}\right]^{\mu_{1}+\mu_{2}} \\
& +k(t)\left[\left(\int_{\Omega} u^{2 q+m+1} d x\right)^{\frac{\mu_{4}}{\mu_{3}+\mu_{4}}}\left(\int_{\Omega} v^{2 p+n+1} d x\right)^{\frac{\mu_{3}}{\mu_{3}+\mu_{4}}}\right]^{\mu_{3}+\mu_{4}} \\
\leq & k(t)\left[\Gamma(t)^{\mu_{1}+\mu_{2}}+\Gamma(t)^{\mu_{3}+\mu_{4}}\right],
\end{aligned}
$$

where

$$
\begin{aligned}
k(t)= & \max \left\{(2 q+m+1) \frac{2 q+7 m+1}{2(4 q+5 m-1)} \varepsilon_{1}^{-\frac{3(2 q+m-1)}{2 q+7 m+1}}|\Omega|^{1-\frac{2 p}{2 p+n+1}} f_{1}(t)^{\frac{2(4 q+5 m-1)}{2 q+7 m+1}},\right. \\
& \left.(2 p+n+1) \frac{2 p+7 n+1}{2(4 p+5 n-1)} \varepsilon^{-\frac{3(2 p+n-1)}{2 p+7 n+1}}|\Omega|^{1-\frac{2 q}{2 q+m+1}} f_{2}(t)^{\frac{2(4 p+5 n-1)}{2 p+7 n+1}}\right\}
\end{aligned}
$$

and

$$
\begin{array}{ll}
\mu_{1}=\frac{2 q+4 m}{2 q+7 m+1}, & \mu_{2}=\frac{2 q(4 p+5 n-1)}{(2 q+m+1)(2 p+7 n+1)} \\
\mu_{3}=\frac{2 p+4 n}{2 p+7 n+1}, & \mu_{4}=\frac{2 p(4 q+5 m-1)}{(2 p+n+1)(2 q+7 m+1)} .
\end{array}
$$


Suppose that

$$
\lim _{t \rightarrow T} \Gamma(t)=+\infty \quad(0<T<+\infty),
$$

then there exists a $t_{1}>0$ such that $\Gamma(t)>1\left(t>t_{1}\right)$.

Integrating (5.7) from $t_{1}$ to $T$, we obtain

$$
\int_{1}^{+\infty} \frac{d \Gamma}{\Gamma^{\mu_{1}+\mu_{2}}+\Gamma^{\mu_{3}+\mu_{4}}} \leq \int_{t_{1}}^{T} k(t) d t \leq \int_{0}^{T} k(t) d t .
$$

Let $\Theta(T)=\int_{0}^{T} k(t) d t$. Obviously, $\Theta(T)$ is increasing. Hence, one has

$$
T \geq \Theta^{-1}(\omega):=T_{0}>0,
$$

where $\omega=\int_{1}^{+\infty} \frac{d \Gamma}{\Gamma^{\mu_{1}+\mu_{2}}+\Gamma^{\mu_{3}+\mu_{4}}}, \Theta^{-1}$ is the inverse function of $\Theta$.

Remark 5.1 The results in Theorem 1.4 still hold for the two-dimensional case. The lower bound estimate to the blow-up time is valid without the convex condition on the domain $\Omega$.

\section{Competing interests}

The authors declare that they have no competing interests.

\section{Authors' contributions}

The authors contributed equally and significantly in this paper.

\section{Author details}

'School of Science, Xihua University, Chengdu, 610039, P.R. China. ${ }^{2}$ Department of Mathematics, Sichuan University, Chengdu, 610065, P.R. China. ${ }^{3}$ Sichuan Sanhe College of Professionals, Luzhou, 646200, P.R. China. ${ }^{4}$ Business School, Sichuan University, Chengdu, 610065, P.R. China.

\section{Acknowledgements}

The authors would like to thank the referees for giving many helpful comments to improve our manuscript. This work is supported by Sichuan Social Science Foundation (SC14TJ06).

Received: 20 September 2015 Accepted: 28 January 2016 Published online: 06 February 2016

\section{References}

1. Bandle, C, Brunner, H: Blow-up in diffusion equations. J. Comput. Appl. Math. 97, 3-22 (1998)

2. Quittner, P, Souplet, P: Superlinear Parabolic Problems. Blow-up, Global Existence and Steady States. Birkhäuser, Basel (2007)

3. Payne, LE, Philippin, GA: Blow-up phenomena in parabolic problems with time dependent coefficients under Dirichlet boundary conditions. Proc. Am. Math. Soc. 141(7), 2309-2318 (2013)

4. Du, LL, Mu, CL, Fan, MS: Global existence and non-existence for a quasilinear degenerate parabolic system with non-local source. Dyn. Syst. 20, 401-412 (2005)

5. Du, LL: Blow-up for a degenerate reaction-diffusion system with nonlinear localized sources. J. Math. Anal. Appl. 324, 304-320 (2006)

6. Du, LL: Blow-up for a degenerate reaction-diffusion system with nonlinear nonlocal sources. J. Comput. Appl. Math. $202,237-247$ (2007)

7. $\mathrm{Du}, \mathrm{LL}, \mathrm{Mu}, \mathrm{CL}$ : Global existence and blow-up analysis to a degenerate reaction-diffusion system with nonlinea memory. Nonlinear Anal., Real World Appl. 9, 303-315 (2008)

8. Fan, MS, Mu, CL, Du, LL: Uniform blow-up profiles for a nonlocal degenerate parabolic system. Appl. Math. Sci. 1, 13-23 (2007)

9. Deng, WB: Global existence and finite time blow up for a degenerate reaction-diffusion system. Nonlinear Anal. 60, 977-991 (2005)

10. Friedman, A, Mcleod, B: Blow-up of solutions of nonlinear degenerate parabolic equations. Arch. Ration. Mech. Anal. 96(1), 55-80 (1986)

11. Fujita, $\mathrm{H}$ : On the blowing up of solutions of the Cauchy problem for $u_{t}=\Delta u+u^{1+\alpha}$. J. Fac. Sci., Univ. Tokyo, Sect. $1 \mathrm{~A}$, Math. 113, 109-124 (1966)

12. Hernndez, J, Mancebo, F, Vega, J: Positive solutions for singular nonlinear elliptic equation. Proc. R. Soc. Edinb., Sect. A Math. 137(1), 41-62 (2007) 
13. Li, FC, Xie, CH: Global existence and blow-up for a nonlinear porous medium equation. Appl. Math. Lett. 16, 185-192 (2003)

14. Payne, LE, Schaefer, PW: Lower bound for blow-up time in parabolic system under Dirichlet conditions. J. Math. Anal. Appl. 328, 1196-1205 (2007)

15. Payne, LE, Philippin, GA: Blow-up phenomena for a class of parabolic system with time dependent coefficients. Appl. Math. 3, 325-330 (2012)

16. Weissler, FB: Existence and nonexistence of global solutions for a heat equation. Isr. J. Math. 38(1-2), 29-40 (1981)

17. Weigner, M: A degenerate diffusion equation with a nonlinear source term. Nonlinear Anal. TMA 28, 1977-1995 (1997)

Submit your manuscript to a SpringerOpen ${ }^{\circ}$ journal and benefit from:

- Convenient online submission

Rigorous peer review

- Immediate publication on acceptance

- Open access: articles freely available online

- High visibility within the field

- Retaining the copyright to your article 